\title{
Fatores de Risco para o Desenvolvimento de Sepse Neonatal Precoce em Hospital da Rede Pública do Brasil ${ }^{*}$
}

\author{
Risk Factors for Early-Onset Neonatal Sepsis in Brazilian Public Hospital \\ Short-Title: Early-Onset Neonatal Sepsis
}

Ana Paula Goulart', Caroline Fraga Valle' ${ }^{1}$, Felipe Dal-Pizzol ${ }^{2}$, Ana Carolina Labor Cancelier ${ }^{3}$.

\section{RESUMO}

JUSTIFICATIVA E OBJETIVOS: O conhecimento dos fatores de risco associados à sepse neonatal precoce em unidade de neonatologia, inserida na realidade de nosso sistema de saúde, no sentido de se detectar, prevenir e adotar medidas específicas e reduzir as taxas de mortalidade nessa faixa etária. O objetivo deste estudo foi determinar os fatores de risco associados a sepse neonatal precoce em hospital de referência em neonatologia ligado à rede pública de saúde.

MÉTODO: Foi realizado um estudo observacional, prospectivo, tipo caso-controle. Foram incluídos os recém-nascidos com diagnóstico de sepse precoce e como controle, recém-nascidos sem infecção neonatal nascido na mesma data do recém-nascido considerado como caso. Foram incluídos 50 casos e três controles para cada caso, resultando em amostra total de 200 pacientes. Foi considerada estatisticamente significativa a associação quando $p<0,05$.

1. Graduanda em Medicina da Universidade do Sul Catarinense, Tubarão, SC.

2. Professor Titular de Medicina, Universidade do Extremo Sul Catarinense, $\mathrm{PhD}$, Criciúma, SC.

3. Professora de Medicina, Universidade do Sul Catarinense, Chefe da UTI Pediátrica - Unidade de Neonatologia, Hospital Nossa Senhora Conceição, Tubarão, SC.

*Recebido do Laboratório de Fisiopatologia Experimental da Universidade Extremo Sul Catarinense (UNESC), Criciúma, SC

Apresentado em 16 de janeiro de 2006

Aprovado para publicação em 08 de maio de 2006

Endereço para correspondência:

Prof. Dr. Felipe Dal-Pizzol

Laboratório de Fisiopatologia Experimental - UNESC

Av. Universitária, 1105 - Bairro Universitário

88006-000 Criciúma, SC

Fax: 554834312759

E-mail: piz@unesc.net - pizzol.ez@terra.com.br

(C)Associação de Medicina Intensiva Brasileira, 2006
RESULTADOS: A freqüência de sepse neonatal precoce no período foi de 50,3 casos para 1000 nascidos vivos. As associações estatisticamente significativas entre os fatores de risco e o desenvolvimento de sepse neonatal precoce foram prematuridade (OR 9,33; $p<0,001$ ), baixo peso ao nascimento (OR 11,74; $p<$ $0,001)$, presença de infecção materna (OR 2,28; $p=$ 0,009 ), filho anterior com infecção neonatal (OR 6,43; $p=0,035)$ e ruptura de membranas mais de 18 horas antes do nascimento (OR 9,33; $p=0,001)$.

CONCLUSÕES: A freqüência de sepse neonatal precoce foi elevada no período do estudo. A prematuridade, o baixo peso ao nascimento, a infecção materna e a ruptura prolongada de membranas são fatores de risco estatisticamente significativos para sepse neonatal precoce.

Unitermos: Fatores de risco, Sepse neonatal precoce

\section{SUMMARY}

BACKGROUND AND OBJECTIVES: The determination of the risk factors to early-onset neonatal sepsis in our country is essential to prevent and reduce the mortality associated with this syndrome. Thus, the objective of this study was to determine the frequency and associated risk factors to early-onset neonatal sepsis in public hospital in Southern Brazil.

METHODS: Observational, case-control study. Were included neonates with diagnostic of early-onset neonatal sepsis and as controls, neonates without neonatal infection. Were included 50 cases and 3 controls for each case resulting in a total sample of 200 patients. Associations were considered significant when $p<$ 0.05 .

RESULTS: The sepsis frequency was 50.3 per 1000 born-alive. Risk factors associated to the development of neonatal sepsis were prematurity (OR 9.33; $p$ $<0.001$ ), low birth weight (OR 11.74; $p<0.001$ ), maternal infection (OR 2.28; $p=0.009$ ), mother with history of previous infant with neonatal sepsis (OR 6.43; $p$ 
$=0.035)$ and rupture of the membranes more than 18 hours before delivery (OR 9.33; $p=0.001$ ).

CONCLUSIONS: Neonatal sepsis was very frequent in the study. Prematurity, low birth weight, maternal infection and mother's having had a previous infant with neonatal sepsis are risk factors for early-onset neonatal sepsis.

Key Words: Early-onset neonatal sepsis, Risk factors

\section{INTRODUÇÃO}

A mortalidade infantil é vista atualmente como um bom indicador de qualidade de vida e dos serviços de saúde em geral. Esse coeficiente vem sofrendo decréscimos na faixa acima do período neonatal como resultado de boas campanhas de vacinação, incentivo ao aleitamento materno e divulgação de medidas preventivas de doenças infecciosas. Entretanto, o componente neonatal da mortalidade infantil engloba relações entre serviços de saúde mais complexos de se manipular, o que dificulta redução mais significativa da mortalidade dessa faixa etária. Estima-se que $50 \%$ dos óbitos no primeiro ano de vida ocorram na primeira semana, o que se denomina período neonatal precoce, números estes que têm chamado atenção dos pesquisadores para causas e formas de prevenção ${ }^{1-3}$.

A sepse neonatal precoce pode ser definida como uma infecção sistêmica que se apresenta até 72 horas após o nascimento, caracterizada por alterações clínicas e laboratoriais diversas ${ }^{3}$. Habitualmente, observase maior ocorrência de sepse no primeiro dia de vida, através de sinais clínicos inespecíficos ${ }^{4-6}$. Nos países em desenvolvimento, a sepse neonatal alcança níveis de até 15,4 casos para cada 1000 nascidos vivos, enquanto que nos Estados Unidos a incidência varia de um a cinco para cada 1000 nascidos vivos ${ }^{7-10}$. Neste último, o Streptococcus do grupo B (SGB) é a bactéria mais comum envolvida na etiologia da sepse neonatal precoce, sendo responsável por aproximadamente 6000 casos por ano $8,11,12$.

$\mathrm{Na}$ literatura existem fatores de risco documentados para a sepse neonatal, os quais podem ser agrupados em fatores maternos, neonatais ou ambientais. Dentre eles se destacam trabalho de parto prematuro, ruptura de membranas mais de 18 horas antes do parto, coIonização materna pelo SGB, febre materna $\left(\geq 38^{\circ} \mathrm{C}\right)$ durante ou imediatamente após o trabalho de parto, sexo masculino, baixo peso ao nascimento $(<2500 \mathrm{~g})$, corioamnionite e filho anterior com infecção neonatal ${ }^{8,11-13}$. Apesar disto, a grande maioria destes estudos são realizados em grandes centros, onde a profilaxia para SBG é rotina, o que não pode ser considerado a realidade de diversos hospitais materno-infantis do Brasil.

Isto justifica a importância do conhecimento dos fatores de risco associados à sepse neonatal precoce em unidade de neonatologia inserida na realidade de nosso sistema de saúde, no sentido de se detectar aqueles passíveis de prevenção, para que se possam adotar medidas específicas e reduzir as taxas de mortalidade nessa faixa etária.

O objetivo deste estudo foi determinar os fatores de risco associados à sepse neonatal precoce em um hospital referência em neonatologia ligado à rede pública de saúde.

\section{MÉTODO}

Após a aprovação pela Comissão de Ética em Pesquisa da Universidade do Sul Catarinense (CEP - UNISUL), foi realizado um estudo observacional, prospectivo, tipo caso e controle, na unidade de neonatologia (UTIN) do Hospital Nossa Senhora da Conceição (HNSC), Tubarão, SC, Brasil. Esta unidade é única na região sul de Santa Catarina, abrangendo uma população de aproximadamente um milhão de habitantes.

Todas as mães dos recém-nascidos incluídos no estudo assinaram o termo de consentimento livre e esclarecido.

Apesar da dificuldade na uniformização do diagnóstico de sepse neonatal ${ }^{14}$, o seu diagnóstico foi baseado nas recomendações da Society for Critical Care Medicine adaptadas para crianças ${ }^{14}$. O tamanho da amostra foi calculado estimando-se o número necessário de paciente (casos e controles) para encontrar uma razão de chances (OR) de ocorrência do evento nos expostos frente aos não expostos de pelo menos 2,5 (OR $=2,5$ ), com confiança de $90 \%$ e poder de $80 \%$, para uma freqüência esperada de $50 \%$ da exposição entre os controles. Foram calculados como sendo necessários 48 casos e três controles para cada caso, resultando em amostra total de 192 pacientes (48 casos e 144 controles). Participaram do estudo, recém-nascidos com sepse precoce, internados na UTIN do HNSC no período de março a agosto de 2004. Para o grupo controle foram selecionados aleatoriamente os recém nascidos sem o diagnóstico de sepse precoce, com estadia no alojamento conjunto do Centro Materno-Infantil do mesmo hospital no período já citado. As variáveis coletadas dos casos e controles relacionadas com 
o desenvolvimento de sepse neonatal precoce analisada foram peso ao nascer, idade gestacional, sexo, idade materna, número de consultas realizadas no prénatal, realização de cultura vaginal para identificação de SGB, resultado de cultura vaginal, história materna de infecção, tempo de ruptura de membranas, tipo de parto, presença de febre (temperatura axilar igual ou maior a $38^{\circ} \mathrm{C}$ ) materna durante o trabalho de parto, antibioticoterapia e profilaxia intraparto e presença de filho anterior com infecção neonatal (relatado pela mãe). Para análise estatística as idades gestacionais foram agrupadas em $\leq 28$ semanas, de 29 a 34 semanas, de 35 a 37 semanas e > 37 semanas completas; os pesos foram agrupados em $\leq 1000$ gramas, 1001 a 1499 gramas, 1500 a 2499 gramas e $\geq 2500$ gramas; as idades maternas foram agrupadas em $\leq 15$ anos, 16 a 25 anos, 26 a 34 anos e $\geq 35$ anos; o número de consultas pré-natal foi agrupado em $<6$ consultas e $\geq 6$ consultas; o tempo de ruptura de membranas foi agrupado em $\leq 18$ ou $>18$ horas.

A existência de associações entre as variáveis independentes de interesse e a ocorrência de sepse foi avaliada pelo teste Qui-quadrado, considerando significativa uma associação com valor de $p<0,05$. Quando pertinente, foi verificada a existência de relação linear para diferentes níveis de uma variável quantitativa entre duas categorias de exposição, através do teste Qui-quadrado para tendência linear.

A compilação dos dados foi feita através do programa EpiData e analisados através do programa Epi-Info 6.04.

\section{RESULTADOS}

O presente estudo englobou 52 casos de sepse neonatal precoce ocorridos na UTIN do HNSC, no período de 16 de março a 18 de agosto de 2004. Do total de casos, dois foram excluídos por não terem seus dados coletados por alta materna. A amostra total resultante foi de 200 pacientes, sendo 50 casos e 150 controles. O número de nascimentos no período foi de 1034 , sendo assim, o total de pacientes representou $19,3 \%$ dos nascidos vivos no período, enquanto os casos representam a totalidade de sepse no período. A freqüência de sepse neonatal precoce no período foi de 50,3 casos para 1000 nascidos vivos.

Eram do sexo masculino $59,5 \%$ do total da amostra e $60 \%$ dos pacientes com sepse. A associação da variável sexo com sepse precoce não mostraram diferença estatística significativa, com odds ratio de 1,03 ( $\mathrm{IC}_{95 \%}$ $0,51-2,09 ; p=0,535)$.
A idade gestacional variou de 24 a 42 semanas, com média de $37,4 \pm 3,7$. A análise dos dados demonstrou maior taxa de sepse neonatal precoce nos recem-nascidos com idade gestacional entre 29 e 34 semanas, perfazendo um total de $46 \%$, conforme detalhado na figura 1. Observou-se ainda, uma tendência linear entre a idade gestacional e a sepse, em que se constatou probabilidade 34,5 vezes maior dos recém-nascidos entre 29 e 34 semanas de desenvolver sepse em comparação aos recém-nascidos com idade gestacional > 37 semanas completas ( $\chi^{2}$ linear: 58,$\left.94 ; p<0,001\right)$. A análise das idades gestacionais, quando classificadas em pré-termo (<37semanas) e a termo ( $>37$ semanas), revelaram que os recém-nascidos pré-termo apresentaram probabilidade de 9,33 vezes maior de desenvolver sepse neonatal em comparação aos nascidos a termo $\left(\mathrm{IC}_{95 \%} 4,06-21,94 ; \mathrm{p}<0,001\right)$.

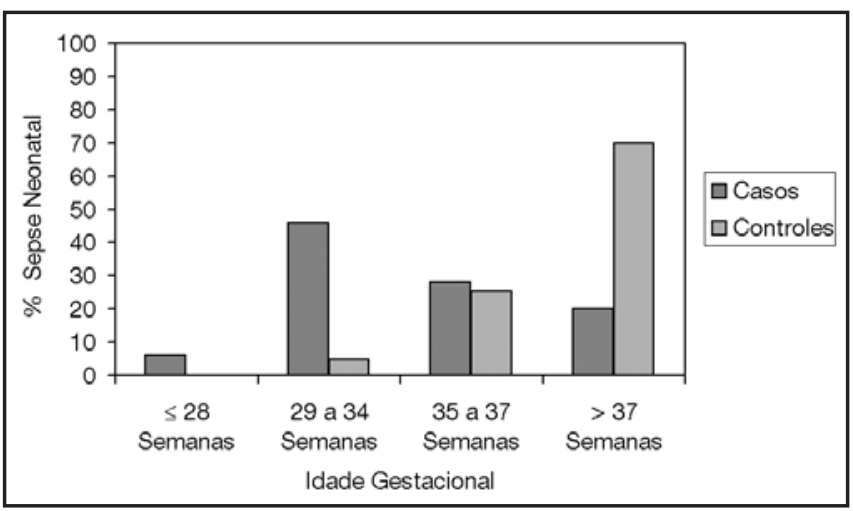

Figura 1 - Distribuição em Porcentagens dos Casos de Sepse Neonatal e Controles Sadios de acordo com a Idade Gestacional. Diferença estatística significativa entre os grupos $p<0,001(n=50$ para grupo sepse neonatal e 150 para os controles sadios).

Ao analisar-se o peso, houve uma variação de 580 a 4450 gramas, com média de $2955,5 \pm 745,7$. Os resultados revelaram que, no período, todos os recémnascidos com peso $\leq 1000 \mathrm{~g}$ desenvolveram sepse neonatal. A taxa de sepse diminuiu nos grupos subseqüentes, conforme representada na figura 2. O teste de tendência linear entre peso e a ocorrência de sepse, demonstraram que os recém-nascidos com peso entre 1001 e $1499 \mathrm{~g}$, tiveram 46,5 vezes mais probabilidade de desenvolver sepse em comparação com aqueles de peso $\geq 2500 \mathrm{~g}$ ( $\chi^{2}$ linear: 46,69; $\left.p<0,001\right)$. Ao comparar os recém-nascidos com peso $<2500 \mathrm{~g}$ aos com $\geq$ $2500 \mathrm{~g}$, observou-se que os primeiros possuem probabilidade de 11,74 vezes maior para desenvolver sepse do que os recém-nascidos com peso $\geq 2500 \mathrm{~g}\left(\mathrm{IC}_{95 \%}\right.$ $5,16-27,04)$. 
Em relação à idade materna, houve uma variação de 14 a 42 anos, com média de $25 \pm 6,9$. Ao analisar a idade das mães de recém-nascidos com sepse, observou-se que $64 \%$ delas apresentaram até 25 anos de idade. A associação desta variável com sepse neonatal precoce não foi estatisticamente significativa $\left(\chi^{2}\right.$ $2,11 ; p=0,551)$. Os dados da associação entre idade materna $\leq 25$ anos e $>25$ anos demonstraram que no primeiro grupo houve probabilidade de 1,4 vezes maior de ocorrer sepse em relação ao segundo grupo $\left(\mathrm{IC}_{95 \%}\right.$ $0,69-2,86)$.

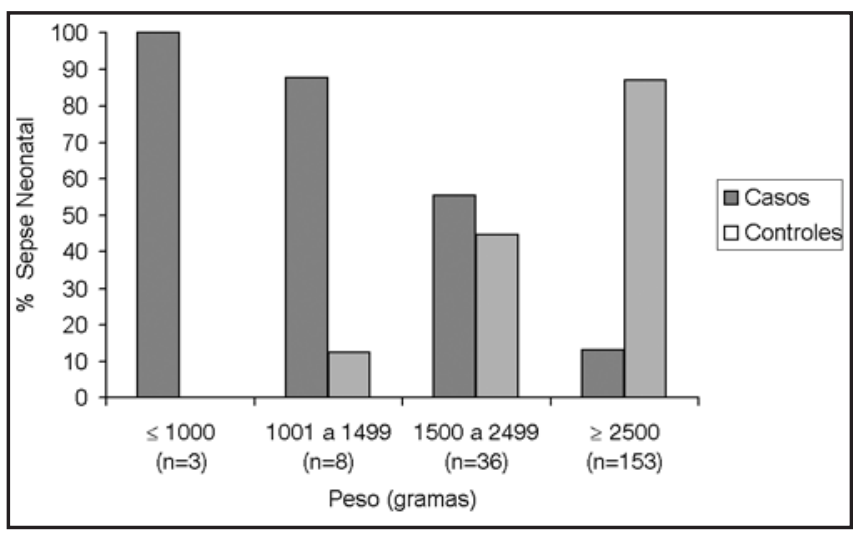

Figura 2 - Distribuição em Porcentagens dos Casos de Sepse Neonatal e Controles Sadios de acordo com o Peso.

Diferença estatística significativa entre os grupos $p<0,001(n=50$ para grupo sepse neonatal e 150 para os controles sadios).

Quanto ao número de consultas realizadas no pré-natal, houve uma variação de 0 a 20 consultas, com média de $6,9 \pm 2,6$. A probabilidade de apresentar sepse neonatal foi 1,78 vez maior no grupo com número inferior a seis consultas em comparação ao com número igual ou superior $\left(\mathrm{IC}_{95 \%} 0,84-3,77\right)$. Porém, a associação desta variável com sepse, não revelou diferença estatística significativa $(p=0,072)$.

Constatou-se um OR de 2,28 de desenvolver sepse para os recém-nascidos cujas mães apresentaram alguma infecção durante a gestação comparada aos recém-nascidos sem história de infecção materna $\left(\mathrm{IC}_{95 \%}\right.$ $1,12-4,63 ; p=0,009$ ), conforme a figura 3. As infecções maternas mais freqüentes foram as do trato urinário $(62,1 \%)$, vulvovaginites $(24,2 \%)$ e corioamnionite $(4,2 \%)$. No período do estudo todos os recém-nascidos em que as mães apresentaram corioamnionite evoluíram com sepse. Dentre os recém-nascidos estudados, cujas mães que apresentaram infecção do trato urinário $37,3 \%$ tiveram sepse, bem como $34,8 \%$ dos recémnascidos cujas mães apresentaram vulvovaginite.

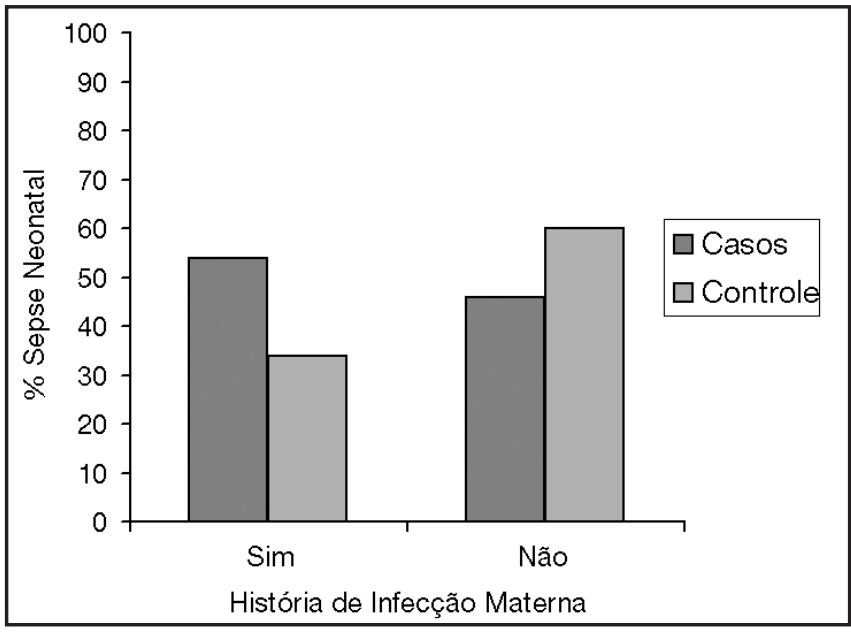

Figura 3 - Porcentagem de Sepse Neonatal entre Casos e Controles de Mães com e sem História de Infecções na Gestação. Diferença estatística significativa entre os grupos $p<0,009(n=50$ para grupo sepse neonatal e 150 para os controles sadios).

$\mathrm{Na}$ amostra, 141 gestantes tiveram ruptura de membranas no ato do nascimento, e 59 apresentaram bolsa rota com variação de 1 hora a 14 dias, sendo a média de 26, $2 \pm 60,5$ horas. Encontrou-se um OR de $9,33\left(\mathrm{IC}_{95 \%} 2,12-46,73 ; \mathrm{p}=0,001\right)$ para a ocorrência de sepse entre os recém-nascidos com bolsa rota havia mais de 18 horas. Dentre os recém-nascidos cujas mães tiveram bolsa rota com mais de 18 horas, $72,7 \%$ foram casos.

Quanto ao tipo de parto, 62,5\% dos bebês nasceram através de cesariana, $30 \%$ por parto vaginal e 7,5\% de parto vaginal com analgesia. A análise estatística não foi significativa quando associada tal variável a sepse $\left(\chi^{2}=1,45 ; p=0,485\right)$. Ao agrupar parto vaginal, com e sem analgesia, e verificar essa associação com apenas duas categorias (cesariana e parto vaginal), encontrou-se OR de $1,03\left(\mathrm{IC}_{95 \%} 0,5-2,09\right)$ para a ocorrência de sepse entre os recém-nascidos de parto vaginal. Além disto, a associação entre febre materna durante o trabalho de parto e a ocorrência de sepse não revelou estatística significativa $(p=0,061)$.

Os resultados mostraram que $66,7 \%$ dos recémnascidos, em que as mães em gestação anterior tiveram filho com infecção neonatal desenvolveram sepse (Figura 4). Os recém-nascidos, cujas mães tinham filho anterior com infecção neonatal tiveram probabilidade de 6,43 vezes maior de desenvolver sepse em comparação aos que não possuíam mães com filho anterior com infecção neonatal $\left(\mathrm{IC}_{95 \%} 0,96\right.$ $-53,24 ; p=0,035)$. 


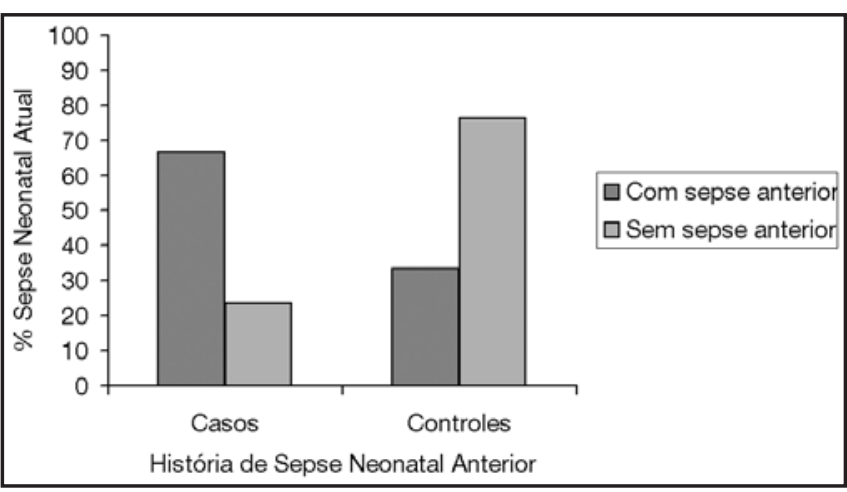

Figura 4 - Porcentagem de Sepse Neonatal entre Casos e Controles com e sem História Anterior de Sepse Neonatal.

Diferença estatística significativa entre os grupos $p<0,001$ ( $n=50$ para grupo sepse neonatal e 150 para os controles sadios).

$\mathrm{Na}$ amostra total $96 \%$ das mães entrevistadas não realizaram o exame de cultura vaginal para SBG. Esta variável não apresentou estatística significativa quando associada a sepse $(p=0,263)$. Das quatro mães que haviam realizado a cultura, três tiveram resultado negativo e uma não soube informar. Dois dos recémnascidos cujos resultados de cultura materna foram negativos, apresentaram sepse.

A antibioticoprofilaxia intraparto foi realizada em apenas nove das 200 gestantes, correspondendo a 4,5\% do total da amostra. Das pacientes estudadas que receberam antibiótico profilático $88,9 \%$ evoluíram com sepse. Os recém-nascidos cujas mães receberam quimioprofilaxia apresentaram probabilidade de 28,38 vezes maior de desenvolver sepse $\left(\mathrm{IC}_{95 \%} 3,39\right.$ - 632,74; $\mathrm{p}<0,001)$. Das nove gestantes submetidas à profilaxia $57 \%$ apresentavam corioamnionite, $29 \%$ bolsa rota e $14 \%$ infecção do trato urinário.

\section{DISCUSSÃO}

Neste estudo foram determinados os fatores de risco para sepse neonatal precoce em Unidade de Neonatologia inserida na realidade da saúde pública de nosso país, que podem diferir grandemente dos fatores descritos em estudos realizados em outras realidades de saúde.

A prematuridade representou ser um importante fator de risco para infecção neonatal precoce em comparação aos recém-nascidos a termo. Benitz e col. encontraram probabilidade de 32,1 vezes maior para os recém-nascidos com menos de 28 semanas de desenvolver sepse em relação aos recém-nascidos com idade gestacional $\geq 37$ semanas $^{15}$. Oddie e Embleton determinaram um
OR de 33,6 de apresentar sepse para os recém-nascidos com até 34 semanas $^{12}$. Assim como prematuridade, baixo peso ao nascimento também ocasionou um substancial aumento do risco de infecção neonatal precoce. Benitz e col. determinaram um OR de 24,8 para tais recém-nascidos desenvolverem sepse em comparação aos com peso $>2500$ gramas $^{15}$.

Quanto ao número de consultas, o que chamou a atenção foi que diferentemente do senso comum, a análise da média $(6,88)$, e da moda $(7,00)$, permitiu afirmar que, apesar dos extremos, a maioria das gestantes realizou o acompanhamento pré-natal com número de consultas próximo ao preconizado pela Organização Mundial de Saúde (OMS), não tendo este fator relação significativa com o desenvolvimento de sepse neonatal precoce. Entretanto, a garantia de que todas as gestantes realizem pelo menos seis consultas de pré-natal ainda é fator a ser atingido para a melhoria da mortalidade infantil.

As infecções maternas, principalmente as do trato genito-urinário, revelaram-se, como na literatura, fatores importantes na elevação do risco de sepse neonatal ${ }^{3,8,11}$. Pôde-se constatar um aumento da ocorrência de sepse nos pacientes cujas mães apresentaram alguma infecção durante o período gestacional, sem relação com a época que esta ocorreu, sendo a infecção mais prevalente a de trato urinário $(62,1 \%)$. A bacteriúria materna por SGB durante a gestação também é um fator reconhecido como causador do aumento do risco de doença invasiva por este patógeno ${ }^{8,11}$. A freqüência de sepse observada entre os recém-nascidos de mães com ruptura de membranas superior a 18 horas, é de aproximadamente $72,7 \%$. Alguns autores relataram aumento ainda maior do risco quando associados sinais e sintomas de corioamnionite, como por exemplo, febre materna intraparto, taquicardia fetal, hipotonia uterina e leucocitose materna ${ }^{10,13}$. Estima-se que a ruptura prematura de membranas amnióticas ocorra em aproximadamente $20 \%$ a $25 \%$ das gestações, sendo mais comum em mulheres colonizadas por $\mathrm{SGB}^{8,12}$.

Outro fator de risco evidente neste estudo, por ocasionar um aumento considerável da probabilidade de sepse quando referido pela mãe, foi ter filho anterior com infecção neonatal. Na literatura existem alguns relatos de que gestação prévia onde o recém-nascido apresentou infecção neonatal, principalmente se ocasionada por SGB, constituiu um fator de risco importante para sepse ${ }^{16,17}$. A associação entre colonização vaginal materna e SGB também é amplamente reconhecida como fator de risco para sepse neonatal por este pa- 
tógeno ${ }^{11,16,17}$. Este risco se eleva quando é associado à ruptura prematura de membranas, febre materna ou prematuridade ${ }^{12}$. Baseado nisso, é recomendado o screening para todas as gestantes entre 35 e 37 semanas de idade gestacional, através de culturas vaginal e retal ${ }^{8}$. Nos programas de assistência pré-natal local essa prática ainda não é rotineira, visto que apenas $2 \%$ das gestantes realizaram o screening através da cultura vaginal, inviabilizando desta forma uma análise mais detalhada acerca dos números reais de colonização por SGB e reforçando a necessidade desta prática no sistema público de saúde.

O emprego da quimioprofilaxia intraparto pode resultar em redução de até $65 \%$ dos casos de sepse neonatal precoce por SGB ${ }^{8,15-17}$. Existem relatos de que a administração de antibiótico profilático intraparto, baseado na presença de fatores de riscos maternos, sem referência à colonização materna por SGB, foi efetiva em $86 \%$ dos casos de prevenção de doença invasiva por $\mathrm{SGB}^{18-20}$. No presente estudo, um número muito restrito da população estudada recebeu a quimioprofilaxia, visto não ser uma prática nos serviços de saúde local. Das parturientes que receberam a quimioprofilaxia, $88,9 \%$ tiveram filhos com infecção neonatal precoce. Não se pode afirmar que tal estratégia não seja eficaz, pois não há dados a respeito da colonização materna e dos agentes mais prevalentes na região.

Sendo a sepse neonatal uma das causas que mais contribuem na taxa de mortalidade no período, tornase cada vez mais necessária a realização de estudos que demonstrem a realidade nacional a esse respeito, no que concerne principalmente a agentes etiológicos mais prevalentes e fatores de risco, para que se possa estabelecer planos de ação na tentativa de reduzir as taxas de infecção neonatal precoce. Os resultados deste estudo sugerem que além da garantia do acesso ao pré-natal conforme recomendado pela OMS, o screening para determinação de colonização materna por SGB e a quimioprofilaxia intraparto devem ser fatores a serem abordados como medida de saúde pública para redução da incidência de sepse neonatal precoce.

\section{REFERÊNCIAS}

01. Araújo BF, Bozzetti MC, Tanaka ACA - Mortalidade neonatal precoce no município de Caxias do Sul: um estudo de coorte. J Pediatr, 2000;76:200-206.

02. Leone CR, Costa MTZ, Kahhale S - Mortalidade Perinatal e Neonatal, em: Marcondes E, Vaz CAF, Ramos ALJ et al - Pediatria Geral e Neonatal, 9th Ed, São Paulo: Sarvier; 2003;252-255.

03. Krebs VLJ, Ceccon MEJR, Feferbaum R - Sepse no Período Neonatal, em: Marcondes E, Vaz CAF, Ramos ALJ et al - Pediatria Geral e Beonatal, 9th Ed, São Paulo: Sarvier; 2003; 560-568.

04. Aggarwal R, Sarkar N, Deorari AK et al - Sepsis in the newborn. Indian J Pediatr, 2001;68:1143-1147.

05. Prociany RS - Avanços no Diagnóstico e Tratamento da Infecção no Recém-Nascido, em: Margotto PR - Resumo de Conferência do $3^{\circ} \mathrm{Sim}-$ pósio Internacional de Neonatologia, Rio de Janeiro, 2002;31.

06. Valle CF, Goulart AP, Dal-Pizzol F et al - Clinical and laboratorial alterations in the diagnosis of neonatal sepsis. RBTI, 2005;17:194-197.

07. Karlowicz MG, Buescher ES, Surka AE - Fulminant late-onset sepsis in a Neonatal Intensive Care Unit, 1988-1997, and the impact of avoiding empiric vancomycin therapy. Pediatrics, 2000;106:1387-1390.

08. Eschenbach DA - Prevention of neonatal group B streptococcal infection. N Engl J Med, 2002;25:347:280-281.

09. Weber MAR, Candiani CL, Garcia JLA et al - Morbidity and mortality from neonatal sepsis in a tertiary care-level hospital. Salud Publica do México, 2003;45:1-6.

10. Escobar GJ, Li D, Armstrong MA et al - Neonatal sepsis workups in infants $\geq 2000$ grams at birth: A population-based study. Pediatrics, 2000;106:256-263.

11. Benitz WE, Gould JB, Druzin ML - Risk factors for early-onset group B streptococcal sepsis: estimation of odds ratios by critical literature review. Pediatrics, 1999;103:1-14.

12. Oddie S, Embleton ND - Risk factors for early onset neonatal group B streptococcal sepsis: case-control study. BMJ, 2002;325:308.

13. Schuchat A, Zywicki SS, Dinsmoor MJ et al - Risk factors and opportunities for prevention of early-onset neonatal sepsis: a multicenter casecontrol study. Pediatrics, 2000;105:21-26.

14. Goldstein B, Giroir B, Randolph A - International pediatric sepsis consensus conference: definitions for sepsis and organ dysfunction in pediatrics. Pediatr Crit Care Med, 2005;6:2-8

15. Benitz WE - Perinatal treatment to prevent early onset group B streptococcal sepsis. Semin Neonatol, 2002;7:301-14.

16. Baltimore RS, Huie SM, Meek Jl et al - Early-onset neonatal sepsis in the era of group B streptococcal prevention. Pediatrics, 2001;108:10941098.

17. Velaphi S, Siegel JD, Wendel GD et al - Early-onset group B streptococcal infection after a combined maternal and neonatal group B streptococcal chemoprophylaxis strategy. Pediatrics, 2003;111:541-547.

18. Pinhata MMM, Nascimento SD - Infecções neonatais hospitalares. J Pediatr, 2001;77:S81-S96.

19. Schrag SJ, Zywicki S, Farley MM et al - Group B streptococcal disease in the era of intrapartum antibiotic prophylaxis. $\mathrm{N}$ Engl $\mathrm{J} \mathrm{Med,}$ 2000;342:15-20.

20. Rouse DJ, Goldenberg RL, Cliver SP et al - Strategies for the prevention of early-onset neonatal group B streptococcal sepsis: a decision analysis. Obstet Gynecol, 1994;83:483-494. 